\title{
Delirium after a traumatic brain injury: predictors and symptom patterns
}

\author{
This article was published in the following Dove Press journal: \\ Neuropsychiatric Disease and Treatment \\ 14 February 2017 \\ Number of times this article has been viewed
}

\author{
Jutaporn Maneewong' \\ Benchalak Maneeton' \\ Narong Maneeton' \\ Tanat Vaniyapong ${ }^{2}$ \\ Patrinee Traisathit ${ }^{3}$ \\ Natthanidnan Sricharoen ${ }^{3}$ \\ Manit Srisurapanont ${ }^{1}$ \\ 'Department of Psychiatry, \\ ${ }^{2}$ Department of Surgery, Faculty of \\ Medicine, ${ }^{3}$ Department of Statistics, \\ Faculty of Science, Chiang Mai \\ University, Chiang Mai, Thailand
}

Correspondence: Benchalak Maneeton Department of Psychiatry, Faculty of Medicine, Chiang Mai University, I I0 Intawaroros Road, Sriphum, Amphur Muang, Chiang Mai 50200, Thailand

Tel +6653935422

Fax +66 53935426

Email benchalak.maneeton@cmu.ac.th
Background: Delirium in traumatic brain injury (TBI) is common, may be predictable, and has a multifaceted symptom complex. This study aimed to examine: 1) the sum score of Glasgow Coma Scale (GCS) and if its component scores could predict delirium in TBI patients, and 2) the prominent symptoms and their courses over the first days after TBI.

Methods: TBI patients were recruited from neurosurgical ward inpatients. All participants were hospitalized within 24 hours after their TBI. Apart from the sum score of GCS, which was obtained at the emergency department (ED), the Diagnostic and Statistical Manual of Mental Disorders, Fifth Edition, diagnostic criteria for delirium were applied daily. The severity of delirium symptoms was assessed daily using the Delirium Rating Scale - Revised-98 (DRS-R-98).

Results: The participants were 54 TBI patients with a mean GCS score of 12.7 (standard deviation [SD] $=2.9)$. A total of 25 patients $(46.3 \%)$ met the diagnosis of delirium and had a mean age of 36.7 years ( $\mathrm{SD}=14.8)$. Compared with 29 non-delirious patients, 25 delirious patients had a significantly lower mean GCS score $(P=0.04)$, especially a significantly lower verbal component score $(P=0.03)$. Among 18 delirious patients, four symptoms of the DRS-R-98 cognitive domain (orientation, attention, long-term memory, and visuospatial ability) were moderate symptoms (score $\geq 2$ ) at the first day of admission. After follow-up, three cognitive (orientation, attention, and visuospatial ability) and two noncognitive symptoms (lability of affect and motor agitation) rapidly resolved.

Conclusion: Almost half of patients with mild to moderate head injuries may develop delirium in the first 4 days after TBI. Those having a low GCS score, especially the verbal component score, at the ED were likely to have delirium in this period. Most cognitive domains of delirium described in the DRS-R-98 were prominent within the first 4 days of TBI with delirium. Three cognitive and two noncognitive symptoms of delirium decreased significantly.

Keywords: Delirium Rating Scale Revised-98, DRS-R-98, brain injuries, traumatic, noncognitive symptoms, cognitive symptoms, Glasgow Coma Scale score

\section{Background}

Traumatic brain injury (TBI) is a brain injury caused by an external mechanical force severe enough to disrupt normal brain function. This injury may lead to hospitalization, need critical care, and cause adverse long-term outcomes. Each year, 235,000 Americans are hospitalized for nonfatal TBI, and 3.8\% of the population in northern Finland experienced at least one hospitalization due to TBI by 35 years of age. ${ }^{1,2}$ TBI can adversely affect both short-term and long-term cognitive, behavioral/emotional, and physical functioning. After 1 year of TBI, $21.3 \%$ of the patients may have at least one psychiatric disorder. ${ }^{3}$ The impact on a person and his or her family can be devastating.

Delirium in TBI is common and may be predictable. The incidence of delirium is varied among samples, largely due to the different levels of TBI severity. A study 
in 85 patients with less severe TBI revealed that $69.4 \%$ of the sample developed delirium during the hospitalization. ${ }^{4}$ This study also found that cognitive impairment on bedside examination and sleep-wake cycle disturbance might be predictors of delirium in this population. Other findings also suggest that the Glasgow Coma Scale (GCS) score may predict the development of delirium in elderly patients with multiple organ traumas. ${ }^{5}$

Delirium has a multifaceted symptom complex. In a medical record review of 80 patients admitted for rehabilitation following TBI, agitation was observed in $70 \%$ of them for an average of 32 days. ${ }^{6}$ In another study, sleep abnormality was found in $66 \%$ of TBI patients. ${ }^{7}$ The aforementioned findings suggest a dearth of evidence on the delirium following TBI. Only a few predictors of such delirium have been found. It is not yet known if the predictability of GCS score in elderly patients with multiple organ traumas is also applicable in patients with head trauma. No systematic study was found regarding delirium symptoms in this population.

This study was, therefore, carried out to determine if the sum score of GCS and its component scores could predict delirium in this population. In addition, for those developing delirium, we further examined prominent symptoms and their courses over the first days after TBI.

\section{Methods}

\section{Study design and setting}

This was an observational prospective study in TBI patients aged $\geq 18$ years. The patients recruited were inpatients of the neurosurgical ward of Chiang Mai University Hospital, a tertiary-care setting in northern Thailand. The study was conducted from May to September 2015. The present study was approved and monitored by the Ethics Committee of Faculty of Medicine, Chiang Mai University. Written informed consent was obtained from patients without delirium or the relatives of patients with delirium. The informed consent was given after the study details had been fully explained.

\section{Selection criteria}

All patients hospitalized for 24 hours with TBI were evaluated. Both male and female patients were included if they were aged $\geq 18$ years. This study excluded patients who were in a coma (GCS score $\leq 3$ ) or had a history of a psychiatric disorder.

\section{Assessment}

All baseline data were collected on the first day of hospitalization. In addition, sociodemographic data such as age, sex, years of education, and history of alcohol use were collected.

\section{GCS}

The GCS was the first grading scale to assess the consciousness of patients. ${ }^{8}$ The assessment of motor, verbal, and eye responses of the GCS characterizes the level of consciousness. The picture provided by these responses enables comparison both between patients and of changes in patients over time that crucially guides management. The three components can be scored separately or combined in a sum score, ranging from 3 to 15 . An approximately linear relationship exists between decreasing sum scores and increasing mortality in patients with TBI, ${ }^{9}$ and the motor component is a strong predictor of poor outcome in moderate or severe TBI. ${ }^{10,11}$ Our study applied the GCS at the emergency department (ED) and used it for daily assessment to determine the consciousness and the severity of TBIs. Based on three modes of behavior, the score of eye opening ranged from $1=$ no eye response, $2=$ response to pain/pressure, $3=$ response to speech/sound, and $4=$ spontaneous response. The verbal response scores were $1=$ no verbal response, $2=$ incomprehensible speech/sounds, $3=$ inappropriate speech/words, $4=$ confused $\mathrm{speech} /$ conversation, and $5=$ orientated conversation. The motor response scores were $1=$ no motor response, $2=$ stereotyped extension, $3=$ stereotyped flexion, $4=$ normal flexion, $5=$ localizes pain, and $6=$ obeys simple commands. Generally, brain injury is classified as severe, GCS 3-8; moderate, GCS 9-12; and mild, GCS 13-15. Mild brain injuries can result in temporary or permanent neurological symptoms, and a neuroimaging test such as computed tomography (CT) scan or magnetic resonance imaging (MRI) may or may not show evidence of any damage. Moderate and severe brain injuries often result in long-term impairments in cognition (thinking skills), physical skills, and/or emotional/behavioral functioning. ${ }^{8}$

\section{Delirium Rating Scale - Revised-98 (DRS-R-98)}

The presence of delirium was examined daily by using the Diagnostic and Statistical Manual of Mental Disorders, Fifth Edition (DSM-5). ${ }^{12}$ The DRS-R-98 ${ }^{13}$ was used daily to assess the severity of delirious symptoms. The DRS-R-98 noncognitive (items 1-8) and cognitive (items 9-13) subscales scores were also computed. ${ }^{14}$ Eight symptoms of the DRS-R-98 noncognitive domain were sleep-wake cycle disturbance, perceptual disturbances and hallucinations, delusions, lability of affect, language, thought process abnormalities, motor agitation, and motor retardation. Five symptoms of the DRS-R-98 cognitive domain included orientation, attention, 
short-term memory, long-term memory, and visuospatial ability. ${ }^{15}$ The DRS-R-98 scale comprises 16 items with a maximum total scale score of 46 points (includes the three diagnostic items' score of 7 points and a maximum severity score of 39 points). The scores of each symptom range from $0=$ normal behavior(s)/no symptom(s), 1= mild symptom, $2=$ moderate symptom, and $3=$ severe or the most disturbed behavior(s). ${ }^{13,16}$ This study used the DRS-R-98 severity score to assess the clinical symptoms of TBI patients.

\section{Statistical analysis}

Statistical analysis was carried out using SPSS for Windows version 22.0. The delirium groups were those developing delirium in the first days of hospitalization. Sociodemographic differences between the delirium and non-delirium groups were compared using the chi-square tests (for categorical data) and the Student's $t$-tests (for interval data). Betweengroup differences on the summation of the severity score and each domain scores of DRS-R-98 on day 1 and GCS at the ED were determined by using the Mann-Whitney $U$ tests. A total of 13 delirious symptoms assessed by using the DRS-R-98 were taken into consideration. The delirium symptom scores obtained on the first days of hospitalization were plotted to see the symptom patterns. In addition, the mean scores of each symptom obtained on these days were compared using the Friedman tests. All calculated $P$-values were two sided, and statistical significance was set at $P<0.05$.

\section{Results}

Between May and September 2015, 272 patients were screened. A total of 210 patients did not satisfy the inclusion criteria of the study. In all, 62 patients with TBI were evaluated at the ED and transferred to the neurosurgery ward. Of these, seven patients had no DRS-R-98 score and one patient had an incomplete record of demographic data. The data of 54 patients were, therefore, included in the present analysis. The mean GCS total score of this sample was 12.7 (standard deviation [SD] =2.9). Figure 1 shows flow diagram for the study enrollment and completion of delirious patients.

In all, 25 patients $(46.3 \%)$ met the diagnosis of delirium and had a mean age of 36.7 years $(\mathrm{SD}=14.8)$. Of the patients with delirium, 21, two, and two developed delirium symptoms on day 1, day 2, and day 3, respectively. Except the significantly higher DRS-R-98 severity score and two domain scores (noncognitive and cognitive subscores) obtained from delirious patients $(P<0.01)$, the data collected on day 1 showed that delirium and non-delirium patients were not significantly different with respect to age, sex, education,

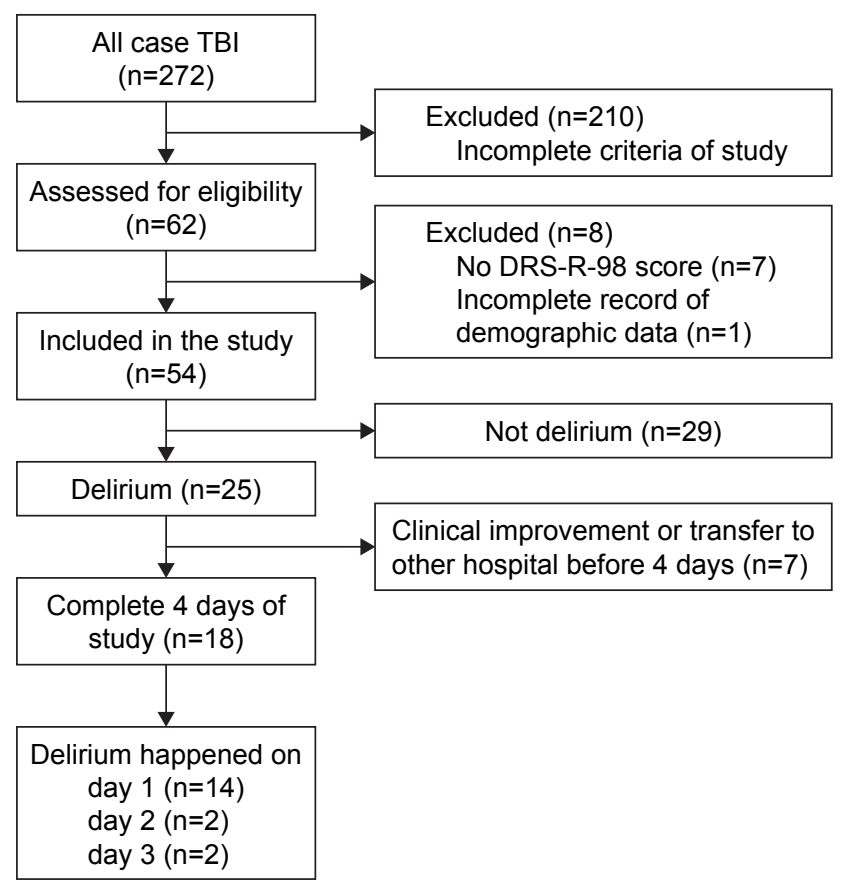

Figure I Flow diagram for the study enrollment and completion of delirious patients. Abbreviations: TBI, traumatic brain injury; DRS-R-98, Delirium Rating Scale Revised-98.

and current alcohol use). Table 1 lists the demographics and clinical characteristics of 54 patients with TBI.

Table 2 lists GCS data at the ED of 54 patients with TBI. The TBI patients with and without delirium had the mean of eye opening scores, verbal response scores, motor response scores, and the sum scores of GCS 2.9 (1.2) vs 3.4 (0.9), 3.4 (1.7) vs 4.2 (1.4), $5.6(0.8)$ vs 5.9 (0.4), and 11.9 (3.2) vs 13.5 (2.4), respectively. From these data, it can be observed that the TBI patients with delirium had not only significantly lower total scores (mean score difference $=1.6, P=0.04$ ) but also significantly lower verbal response subscores (mean score difference $=0.8, P=0.03$ ) than those of the TBI patients without delirium.

Of the 25 delirium participants, 18 patients were hospitalized for at least 4 days, and the data of these 18 patients were included in the study of delirious symptom patterns. Table 3 lists the demographics and clinical characteristics of 18 TBI patients with delirium included in the study of symptom patterns. The mean age of these TBI patients with delirium was 38.9 years (15.5), most of them had less than a primary school education ( $<6$ years) and were males. A total of $\sim 60 \%$ of these TBI patients with delirium were currently using alcohol. All the other data are listed in Table 3. While 14 patients had delirium on day 1 , two patients each developed delirium on day 2 and day 3 . The causes of injury were traffic injury (14 patients, 77.8\%) and falls (four patients, $22.2 \%$ ). During the 4-day observation study, all patients 
Table I Demographics and clinical characteristics of 54 patients with TBI

\begin{tabular}{|c|c|c|c|}
\hline $\begin{array}{l}\text { Demographic and } \\
\text { clinical data }\end{array}$ & $\begin{array}{l}\text { TBI with } \\
\text { delirium }(n=25)\end{array}$ & $\begin{array}{l}\text { TBI without } \\
\text { delirium }(n=29)\end{array}$ & $\begin{array}{l}\text { Statistical test, } \\
P \text {-value }\end{array}$ \\
\hline Age (years), mean (SD) & $36.7(14.8)$ & $36.5(17.1)$ & $T=0.05, P=0.96$ \\
\hline Male, n (\%) & $21(38.9)$ & $18(33.3)$ & $\chi^{2}=3.22, P=0.07$ \\
\hline \multicolumn{4}{|l|}{ Education, n (\%) } \\
\hline$<6$ years & $14(25.9)$ & $12(22.2)$ & $\chi^{2}=1.15, P=0.28$ \\
\hline$\geq 6$ years & II (20.4) & $17(3 \mid .5)$ & \\
\hline Current alcohol use, $\mathrm{n}(\%)$ & $17(3 \mid .5)$ & $21(38.9)$ & $\chi^{2}=0.72, P=0.13$ \\
\hline \multicolumn{4}{|l|}{ Day I, mean (SD) } \\
\hline Severity scores & $16.6(6.7)$ & $6.2(4.3)$ & $U=53.5, P=<0.0 I^{*}$ \\
\hline Noncognitive scores & $6.0(4.5)$ & $2.2(2.2)$ & $U=\mid 49.0, P=<0.01 *$ \\
\hline Cognitive scores & $10.5(2.9)$ & $4.0(2.5)$ & $U=31.0, P=<0.0 I^{*}$ \\
\hline
\end{tabular}

Notes: Chi-square test was used for analysis of categorical data, Student's $t$-test was used for continuous data, and Mann-Whitney $U$ test was used for ordinal data. *Significantly different.

Abbreviations: TBI, traumatic brain injury; SD, standard deviation.

received environmental manipulation to minimize delirium. Two patients also received $0.5 \mathrm{mg} /$ day of risperidone, and one patient received $0.25 \mathrm{mg} /$ day of haloperidol.

Figure 2 shows the mean domain scores of noncognitive and cognitive symptoms of 18 TBI patients with delirium. Both the eight symptoms of the DRS-R-98 noncognitive domain, such as sleep-wake cycle disturbance, perceptual disturbances and hallucinations, and the five symptoms of the DRS-R-98 cognitive domain, for example orientation, attention, and short-term memory, are arranged on the $x$-axis. The mean domain scores of each symptom range from $0=$ normal behavior(s)/no symptom(s) to $3=$ severe or the most disturbed behavior(s), which are labeled on the $y$-axis.

The mean domain scores of the DRS-R-98 noncognitive and cognitive symptoms were highest on day 1 , and most of these scores decreased gradually over the 4 days of observation. On the first day of the study, TBI patients with delirium had prominent four cognitive symptoms from 13 symptoms of DRS-R-98, including orientation (mean $[\mathrm{SD}]=2.1[1.0]$ ), attention (mean $[\mathrm{SD}]=2.1[0.8]$ ), long-term memory (mean $[\mathrm{SD}]=2.6[0.9]$ ), and visuospatial ability (mean $[\mathrm{SD}]=2.9[0.2]$ ).

Figure 2 shows that the mean scores of eight DRS-R-98 noncognitive domains were not prominent. The mean (SD) scores of sleep-wake cycle, lability of affect, language, thought process abnormalities, perceptual disturbances and hallucinations, delusions, motor agitation, and motor retardation were 1.6 (1.1), $1.2(1.1), 1.2(0.9), 1.3(0.9), 0.3(0.6)$, $0.2(0.7), 0.8(1.0)$, and $0.1(0.3)$, respectively.

Comparing the mean scores obtained on the first to the fourth day of study, the Friedman tests showed that three cognitive symptoms, including orientation $\left(\chi^{2}=11.02\right.$, $P=0.01)$, attention $\left(\chi^{2}=10.16, P=0.02\right)$, and visuospatial ability $\left(\chi^{2}=14.91, P=<0.01\right)$, were significantly decreased from the baseline. Based on noncognitive symptoms, only lability of affect $\left(\chi^{2}=13.71, P=<0.01\right)$ and motor agitation $\left(\chi^{2}=12.73\right.$, $P=<0.01)$ were significantly reduced from the baseline.

\section{Discussion}

Given that the GCS classifies a TBI as mild (13-15), moderate (9-12), or severe $(3-8),{ }^{8}$ our sample (mean GCS total score $=12.7, \mathrm{SD}=2.9$ ) consisted of those having a mild to moderate head injury. This study presents the illness course of TBI in the first 4 days. Almost a half of them developed delirium after having head injury. The GCS total and verbal response scores obtained at the ED might predict delirium. At the first day of study, cognitive symptoms of delirium were more severe than noncognitive ones. Delirious symptoms

Table 2 GCS data at the ED of 54 patients with TBI

\begin{tabular}{|c|c|c|c|}
\hline GCS score at the ED & $\begin{array}{l}\text { TBI with } \\
\text { delirium }(n=25)\end{array}$ & $\begin{array}{l}\text { TBI without } \\
\text { delirium }(n=29)\end{array}$ & Statistical test \\
\hline Eye opening (maximum score $=4$ ) & $2.9(1.2)$ & $3.4(0.9)$ & $U=275.0, P=0.10$ \\
\hline Verbal response (maximum score $=5$ ) & $3.4(1.7)$ & $4.2(1.4)$ & $U=25 I .5, P=0.03^{*}$ \\
\hline Motor response (maximum score $=6$ ) & $5.6(0.8)$ & $5.9(0.4)$ & $U=292.5, P=0.09$ \\
\hline The sum score of GCS (maximum score $=15$ ) & $11.9(3.2)$ & $13.5(2.4)$ & $U=274.5, P=0.04^{*}$ \\
\hline
\end{tabular}

Notes: Statistical test: Mann-Whitney $U$ test for analysis of ordinal data. *Significantly different.

Abbreviations: GCS, Glasgow Coma Scale; ED, emergency department; TBI, traumatic brain injury. 
Table 3 Demographics and clinical characteristics of $\mid 8$ TBI patients with delirium included in the study of symptom patterns

\begin{tabular}{|c|c|}
\hline $\begin{array}{l}\text { Demographic and } \\
\text { clinical data }\end{array}$ & $\begin{array}{l}\text { TBI with } \\
\text { delirium }(n=18)\end{array}$ \\
\hline Age (year), mean (SD) & $38.9(15.5)$ \\
\hline Male, n (\%) & $15(83.3)$ \\
\hline \multicolumn{2}{|l|}{ Education, $\mathrm{n}(\%)$} \\
\hline$<6$ years & II (6I.I) \\
\hline$\geq 6$ years & $7(38.9)$ \\
\hline Current alcohol use, n (\%) & $12(66.7)$ \\
\hline The sum score of GCS at ED, mean (SD) & $12.6(2.9)$ \\
\hline The sum score of GCS on day I, mean (SD) & $13.9(1.1)$ \\
\hline \multicolumn{2}{|l|}{ DRS-R-98 on day I, mean (SD) } \\
\hline Severity scores & I8.I (7.2) \\
\hline Noncognitive scores & $6.7(5.0)$ \\
\hline Cognitive scores & II.3 (2.9) \\
\hline
\end{tabular}

Abbreviations: TBI, traumatic brain injury; GCS, Glasgow Coma Scale; ED, emergency department; DRS-R-98, Delirium Rating Scale - Revised-98.

likely to be rapidly resolved were orientation, attention, visuospatial ability, lability of affect, and motor agitation.

Although the prevalence rate of delirium in this study $(46.3 \%)$ was lower than that in a previous study, it still confirms that delirium is common in TBI patients.
Nakase-Thompson et $\mathrm{al}^{4}$ have found delirium in $69.4 \%$ of TBI patients. Apart from other methodological differences, it is possible that the lower prevalence rate was caused by the less severe head injuries in our sample.

The GCS score has been used to predict several outcomes of TBI patients. Previous findings suggest that it may not be used to predict the providing of neurosurgical interventions, ${ }^{17}$ but it is independently associated with 1-year survival rates. ${ }^{18}$ Age and the GCS total score obtained at the ED may predict the development of delirium in elderly patients with multiple organ traumas. ${ }^{5}$ Apart from the confirmation of the predictability of GCS total score, the present findings add more evidence that the GCS verbal response score may be superior to the other two domain scores in predicting delirium in this population.

By using a delirium rating scale, this study systematically and daily assessed delirious symptoms in the first 4 days after having a TBI. Based on the 13 symptoms of DRS-R-98, the cognitive symptoms were more prominent than noncognitive symptoms in TBI patients with delirium at the first day. This phenomenon may be attributed to the fact

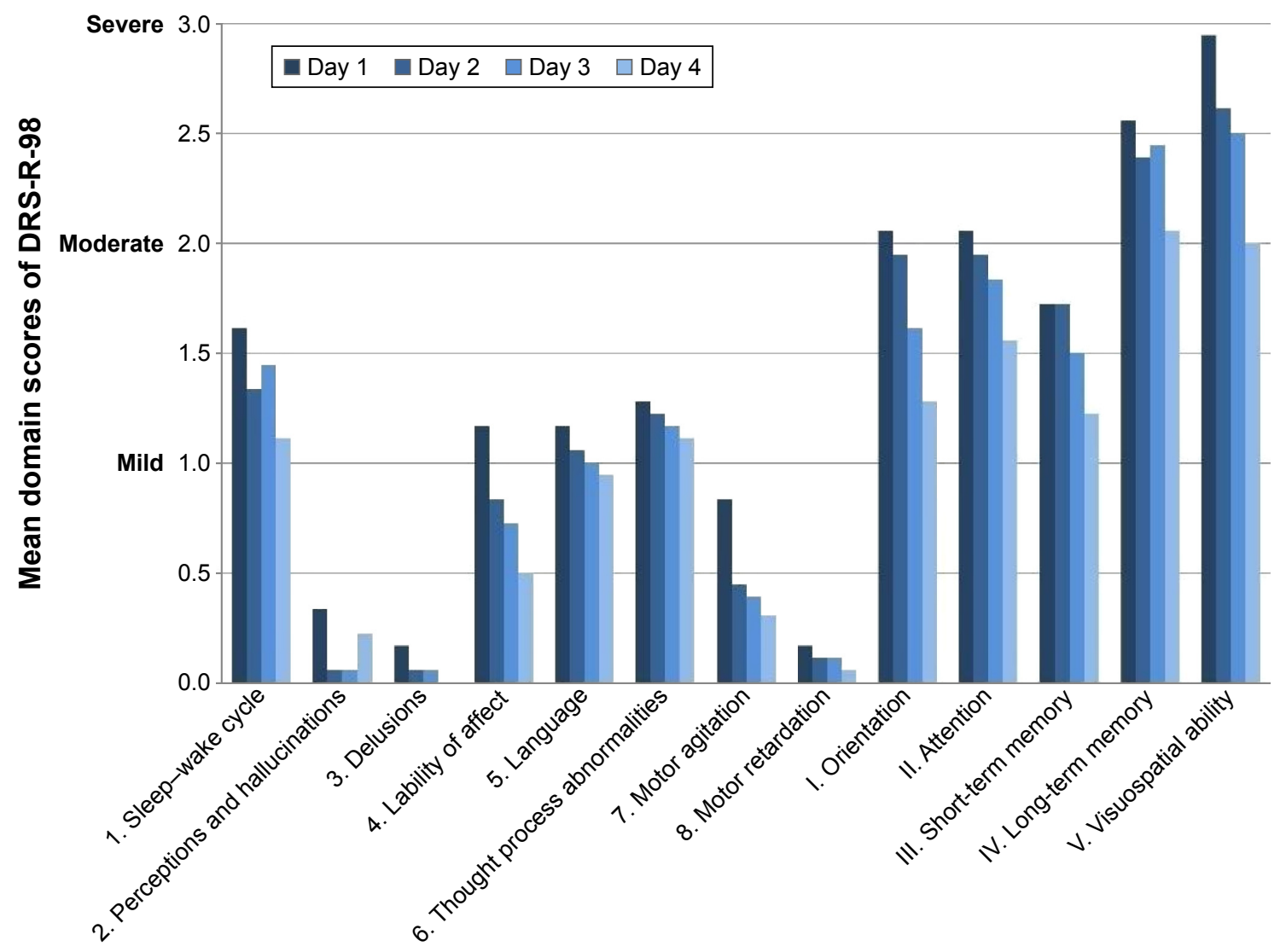

Noncognitive and cognitive domains of DRS-R-98

Figure 2 Mean domain scores of noncognitive and cognitive domains obtained from I8 TBI patients with delirium, from day I to day 4. Abbreviations: TBI, traumatic brain injury; DRS-R-98, Delirium Rating Scale - Revised-98. 
that all subjects had brain insult. After 4 days of follow-up study, the sum score of GCS gradually increased and the sum score of DRS-R-98 gradually decreased. It suggested that the delirious symptoms possibly decreased after the brain gradually recovered. According to cognitive symptoms, orientation, attention, and visuospatial ability appeared to rapidly improve, while two noncognitive symptoms, including lability of affect and motor agitation, resolved on day 4. However, sleep-wake problem was persistent, which was similar to previous evidences. ${ }^{6,7}$

This study had some limitations. First, the sample size was small. Therefore, false-negative findings may not be ruled out. Second, the mean GCS total scores of 11.9 and 13.5 obtained from delirium and non-delirium groups suggested that only a few patients with a severe TBI were included in this study. Third, as a part of the service network, some participants were referred to another hospital or discharged from the hospital within the first 4 days of their hospitalization. Last, some participants had multiple organ injuries. However, other organ injuries, which might also contribute to the development of delirium, were not taken into account.

\section{Conclusion}

Almost a half of patients with mild to moderate head injuries may develop delirium in the first 4 days after a TBI. Those having low GCS total and verbal response scores in the ED are likely to have delirium in this period. Most delirious symptoms described in the DRS-R-98 were prominent within the first day of TBI with delirium. Delirium symptoms that rapidly resolved included orientation, attention, visuospatial ability, lability of affect, and motor agitation. Further research in a larger sample size or with a longer period of observation is warranted.

\section{Acknowledgment}

This work was supported by the CMU Mid-Career Researcher Program, Chiang Mai University, Thailand.

\section{Author contributions}

All authors contributed toward data analysis, drafting and critically revising the paper, gave final approval of the version to be published, and agree to be accountable for all aspects of the work.

\section{Disclosure}

Benchalak Maneeton has been an advisory board member of Pfizer and received honoraria and/or travel reimbursement from Lundbeck, Servier, and Pfizer. Narong Maneeton has received travel reimbursement from Lundbeck and Pfizer. Manit Srisurapanont has been an advisory board member of Lundbeck, Novartis, and Servier and received speaker's honoraria from Lundbeck and Servier and grant/research support from Lundbeck and Servier. Jutaporn Maneewong, Tanat Vaniyapong, Patrinee Traisathit, and Natthanidnan Sricharoen report no conflicts of interest in this work.

\section{References}

1. Adamis D, Rooney S, Meagher D, Mulligan O, McCarthy G. A comparison of delirium diagnosis in elderly medical inpatients using the CAM, DRS-R98, DSM-IV and DSM-5 criteria. Int Psychogeriatr. 2015; 27(6):883-889.

2. Corrigan JD, Selassie AW, Orman JA. The epidemiology of traumatic brain injury. J Head Trauma Rehabil. 2010;25(2):72-80.

3. Deb S, Lyons I, Koutzoukis C, Ali I, McCarthy G. Rate of psychiatric illness 1 year after traumatic brain injury. Am J Psychiatry. 1999;156(3): 374-378.

4. Nakase-Thompson R, Sherer M, Yablon SA, Nick TG, Trzepacz PT. Acute confusion following traumatic brain injury. Brain Inj. 2004; 18(2):131-142.

5. Bryczkowski SB, Lopreiato MC, Yonclas PP, Sacca JJ, Mosenthal AC. Risk factors for delirium in older trauma patients admitted to the surgical intensive care unit. J Trauma Acute Care Surg. 2014;77(6):944-951.

6. Nott MT, Chapparo C, Baguley IJ. Agitation following traumatic brain injury: an Australian sample. Brain Inj. 2006;20(11):1175-1182.

7. Nakase-Richardson R, Sherer M, Barnett SD, et al. Prospective evaluation of the nature, course, and impact of acute sleep abnormality after traumatic brain injury. Arch Phys Med Rehabil. 2013;94(5):875-882.

8. Teasdale G, Jennett B. Assessment of coma and impaired consciousness. A practical scale. Lancet. 1974;2(7872):81-84.

9. Teasdale G, Maas A, Lecky F, Manley G, Stocchetti N, Murray G. The Glasgow Coma Scale at 40 years: standing the test of time. Lancet Neurol. 2014;13(8):844-854.

10. Murray GD, Butcher I, McHugh GS, et al. Multivariable prognostic analysis in traumatic brain injury: results from the IMPACT study. J Neurotrauma. 2007;24(2):329-337.

11. Reith FC, Van den Brande R, Synnot A, Gruen R, Maas AI. The reliability of the Glasgow Coma Scale: a systematic review. Intensive Care Med. 2016;42(1):3-15.

12. Association AP. Diagnostic and Statistical Manual of Mental Disorders. 5th ed. Arlinton, VA: American Psychiatric Association; 2013.

13. Trzepacz PT, Mittal D, Torres R, Kanary K, Norton J, Jimerson N. Validation of the Delirium Rating Scale-revised-98: comparison with the delirium rating scale and the cognitive test for delirium. J Neuropsychiatry Clin Neurosci. 2001;13(2):229-242.

14. Tahir TA, Eeles E, Karapareddy V, et al. A randomized controlled trial of quetiapine versus placebo in the treatment of delirium. J Psychosom Res. 2010;69(5):485-490.

15. Rajlakshmi AK, Mattoo SK, Grover S. Relationship between cognitive and non-cognitive symptoms of delirium. Asian J Psychiatry. 2013;6(2): 106-112.

16. Thurber S, Kishi Y, Trzepacz PT, et al. Confirmatory factor analysis of the Delirium Rating Scale Revised-98 (DRS-R98). J Neuropsychiatry Clin Neurosci. 2015;27(2):e122-e127.

17. Ashkenazi I, Schecter WP, Peleg K, et al. Glasgow Coma Scale Score in survivors of explosion with possible traumatic brain injury in need of neurosurgical intervention. JAMA Surg. 2016;151(10):954-958.

18. Signorini DF, Andrews PJ, Jones PA, Wardlaw JM, Miller JD. Predicting survival using simple clinical variables: a case study in traumatic brain injury. J Neurol Neurosurg Psychiatry. 1999;66(1):20-25. 


\section{Publish your work in this journal}

Neuropsychiatric Disease and Treatment is an international, peerreviewed journal of clinical therapeutics and pharmacology focusing on concise rapid reporting of clinical or pre-clinical studies on a range of neuropsychiatric and neurological disorders. This journal is indexed on PubMed Central, the 'PsycINFO' database and CAS, and is the official journal of The International Neuropsychiatric Association (INA). The manuscript management system is completely online and includes a very quick and fair peer-review system, which is all easy to use. Visit http://www.dovepress.com/testimonials.php to read real quotes from published authors.

\footnotetext{
Submit your manuscript here: http://www.dovepress.com/neuropsychiatric-disease-and-treatment-journal
} 